\title{
Parenté, personne et genre : pour une approche relationnelle des marges
}

\section{Entretien avec Laurence Hérault et Irène Théry}

\author{
Groupe EnCore
}

Émulations - Revue de sciences sociales

2019, n 32, «Aux frontières de la parenté. Un éclairage par les marges ».

\section{Article disponible à l'adresse suivante}

https://ojs.uclouvain.be/index.php/emulations/article/view/encore

\section{Pour citer cet article}

Groupe EnCore, «Parenté, personne et genre : pour une approche relationnelle des marges. Entretien avec Laurence Hérault et Irène Théry », Émulations, n 32 , Mise en ligne le 20 avril 2020.

DOI : 10.14428/emulations.032.08

Distribution électronique : Université catholique de Louvain (Belgique) : ojs.uclouvain.be

(C) Cet article est mis à disposition selon les termes de la Licence Creative Commons Attribution, Pas d'Utilisation Commerciale 4.0 International. http://creativecommons.org/licenses/by-nc/4.0/

Éditeur : Émulations - Revue de sciences sociales / Presses universitaires de Louvain https://ojs.uclouvain.be/index.php/emulations

ISSN électronique : 1784-5734

PUL PRESSES UNIVERSITAIRES DE LOUVAIN 


\title{
Parenté, personne et genre : pour une approche relationnelle des marges
}

\author{
Entretien avec Laurence Hérault et Irène Théry
}

Groupe EnCoRe ${ }^{1}$

\begin{abstract}
[Résumé] Le groupe EnCoRe a réuni Laurence Hérault (professeure à Aix-Marseille Université), anthropologue de la parenté, et Irène Théry (directrice d'études à l'EHESS), sociologue du droit de la famille, pour apporter un éclairage à l'utilisation des notions de marges, confins, seuils et frontières dans l'analyse de la parenté contemporaine. À partir de leurs travaux, cet entretien propose un dialogue sur la mobilisation de la marge comme catégorie analytique, outil descriptif et révélatrice des normes sociales. Les deux chercheuses encouragent une approche relationnelle des marges.
\end{abstract}

Mots-clés : parenté, personne, genre, approche relationnelle, marges.

Kinship, personhood and gender: for a relational approach of margins Interview with Laurence Hérault and Irène Théry

[Abstract] EnCoRe research group gathered Laurence Hérault, kinship anthropologist, and Irène Théry, family law sociologist, to shed light on the use of the notions of margins, edges, thresholds and boundaries in contemporary kinship studies. Based on their research, this interview offers a dialogue about the use of margins as an analytical category, a descriptive tool and an indicator of social norms. Both researchers support a relational approach of margins.

Keywords: kinship, personhood, gender, relational approach, margins.

Le groupe EnCoRe a réuni Laurence Hérault ${ }^{2}$ (professeure à l'Université Aix-Marseille), anthropologue de la parenté, et Irène Théry3 (directrice d'études à l'École des hautes études en sciences sociales), sociologue du droit de la famille, pour apporter un éclairage à l'utilisation des notions de marges, confins, seuils et frontières dans l'analyse de la parenté contemporaine. À partir de leurs travaux, cet entretien propose un dialogue sur la mobilisation de la marge comme catégorie analytique, outil descriptif et révélatrice des normes sociales.

\footnotetext{
${ }^{1}$ Le groupe de recherche EnCoRe (Engendrement, Corps, Relations) est composé d'Anne-Sophie Giraud (Centre nationale de la recherche scientifique, LISST-CAS, France), Léa Linconstant (Aix Marseille Université, IDEMEC, France), Hélène Malmanche (École des hautes études en sciences sociales, CESPRA, France), Anaïs Martin (École des hautes études en sciences sociales, Centre Norbert Elias, France) et Manon Vialle (Centre national de la recherche scientifique, Centre Norbert Elias, France).

${ }^{2}$ Aix Marseille Université, IDEMEC, France.

3 École des hautes études en sciences sociales, Centre Norbert Elias, CESPRA, France.
} 
Groupe EnCoRe : Comment, dans vos travaux, avez-vous rencontré ces questions de frontières, confins, limites et seuils?

Irène Théry: L'enquête que j'ai réalisée pour ma thèse sur les conflits autour des gardes d'enfants lors du divorce et sur la notion de l'intérêt de l'enfant (Théry, 1983) m’a amenée à aborder « l'impossible rôle du second parent ». On s'attendait à la fois à ce que le beau-parent soit une sorte de parent de substitution et en même temps on lui refusait ce rôle. Après avoir fait un état de la question dans la littérature anglo-saxonne en 1987, j'ai mené la première enquête française sur le statut juridique et social du beau-parent en 1990. Il s'agissait de donner une consistance à cette notion de statut marginal, de rôle non défini. L'une des formules que les beaux-parents employaient pour se définir était « je ne suis ni parent ni ami ». Il fallait comprendre ce que cette double négation signifiait concrètement pour les personnes, en les interrogeant précisément, ce qu'elles s'autorisaient à faire ou ne pas faire. C'est là qu'il m'est apparu qu'on ne pouvait pas comprendre l'ici et maintenant sociologique sans une prise en compte de l'héritage culturel sur la longue durée. Longtemps, le beau-parent a été perçu comme «le substitut menaçant du mort ». Dans une société où le veuvage était fréquent et les rôles masculins et féminins par principe très différenciés, le parent veuf ne pouvait pas rester seul, et le remariage était très fréquent. Mais ce remariage, quoiqu'autorisé, était perçu comme une transgression du principe monogamique chrétien. Le beau-parent est un substitut nécessaire, qui pourtant met en cause l'idéal familial ; de là, le stigmate, incarné par les figures de la marâtre et du parâtre.

$\mathrm{Au} \mathrm{XX}^{\mathrm{e}}$ siècle, les premières recompositions après divorce se développent, et le beau-parent est peu à peu valorisé comme «le substitut rassurant du coupable ». Le divorce pour faute, seul autorisé, est vécu comme une sorte de veuvage social purifiant la famille de l'époux fautif : une fois celui-ci éliminé (garde confiée à l'innocent), il faut refaire une famille ressemblant le plus possible à une famille nucléaire classique. Le beau-père adopte les enfants et apparaît comme une figure positive de réintégration dans la normalité sociale de la famille nucléaire.

Enfin, dans les années 1970, avec le développement du divorce de masse, une nouvelle règle sociale émerge lorsque la filiation devient le symbole même du lien inconditionnel et indissoluble, et qu'il n'est plus question qu'un parent divorcé perde son statut de parent. Dès lors, le beau-parent devient une figure parentale additionnelle et sa marginalité prend une nouvelle figure : le « ni parent ni ami » heurte nos représentations collectives, parce qu'il est à la frontière entre deux types de liens sociaux pensés comme opposés l'un à l'autre, la parenté et l'amitié. Le jurisconsulte Domat a théorisé cette opposition dans un traité analysant les différentes sortes de liens humains (1689). La parenté est vraiment le cœur des obligations statutaires, auxquelles l'individu doit se plier quoiqu'il en pense. Le principe de l'amitié, au contraire, est d'être le symbole même du rapport électif libre, sans droits ni devoirs, un lien dont le prix est de n'exister que par la volonté de chaque ami. 
Ce recul historique m’a permis de voir que la marginalité du statut de beau-parent peut prendre des figures successives très différentes, qui vont se déposer dans la mémoire collective, mais que finalement le beau-parent est toujours « l'autre » du statut parental.

Laurence Hérault : J'ai quant à moi rencontré très tôt la question des marges dans mon travail puisque j'ai commencé par travailler sur la question des rituels du cycle de vie dans une région de l'ouest de la France (Hérault, 1996). Je m’intéressais à la façon dont ces cérémonies peuvent se modifier et varier au cours du temps. L'approche d'Arnold van Gennep (1991), et notamment la question de la marge, venait comme un outil d'analyse pour les comprendre. En même temps, je trouvais déjà ce schéma de la « séparation-marge-agrégation » un peu piégeant, enfermant. Il y avait aussi une volonté de critiquer l'approche van gennepienne. Par la suite, dans mon parcours de recherche, je change de thématique : j’abandonne le religieux et je m'intéresse plus particulièrement à la question du genre et à celle de la transidentité (Hérault, 2014). Là je rencontre la question des marges d'une autre façon, comme une qualification sociale. Ce n'est pas moi qui la propose ; ce n'est pas un outil d'analyse. C'est une qualification sociale concernant l'expérience d'un certain nombre de personnes. Et cette qualification sociale est dans le double sens de " marge », de « marginal », c'est-à-dire une expérience qui est quantitativement faible, peu importante en nombre : on peut dire que numériquement ou statistiquement, il y a peu de personnes trans dans nos sociétés. Mais cette notion est aussi utilisée dans un sens peut-être plus problématique, c'est-à-dire que c'est aussi une question de marge du point de vue des normes, parce qu'il y a là une non-conformité aux normes de la sexuation qui généralement est quelque chose de stable, et qui là, ne l'est pas. Cette non-conformité est aussi très souvent, très rapidement, associée à de la disqualification. Cela n'est pas une marginalité qui est forcément positive, et la transidentité apparaît donc comme un lieu de transgression. Il faudrait pouvoir comprendre cette marginalité transgressive et en faire une ethnographie. Pour ma part, ce n'est pas du tout ce qui m’intéressait. Je voulais comprendre comment justement on en faisait quelque chose de marginal, et par quelles procédures.

Par ailleurs, dans l'expérience de la transidentité, c'est le moment de la transition qui a souvent été l'objet privilégié par les sciences sociales et l'approche de van Gennep s'est trouvée réinvestie. L'anthropologue américaine Anne Bolin (1987) s'est intéressée à la transition des femmes trans dans les années 1980, et a repris la notion de marge pour penser la transition. Pour elle, il s'agit d'un rite de passage d'une identité masculine vers une identité féminine, et ce temps-là est, selon elle, celui de la marge entre ces deux identités. J'ai aussi critiqué cette compréhension de la transition car si sa ritualisation permet de saisir assez bien la façon dont nos sociétés comprennent le fait de revendiquer une autre inscription sexuée, elle ne rend pas de façon satisfaisante l'expérience vécue par les personnes trans. 
Groupe EnCoRe: Que nous apprennent les marges sur la norme et quels sont les rapports des unes à l'autre?

Irène Théry : On ne peut pas élucider ces situations marginales, et le stigmate qui les caractérise, si on n’interroge pas la norme. Ainsi, dans un réseau pluridisciplinaire sur les familles recomposées que j'avais impulsé, nous sommes passés collectivement d'une interrogation sur le rapport beau-parent/bel-enfant, à une interrogation sur les limites de la famille et les confins de la parenté. Qu'est-ce qu'une configuration familiale dans laquelle chacun des membres ne définit pas les contours de « ma famille » de la même façon ? Nous sommes ainsi passés du point de vue des adultes (leur foyer) au point de vue de l'enfant (la circulation entre les foyers). Et c'est là que nous avons fait un progrès en comprenant pourquoi, malgré la fréquence des recompositions familiales, le statut beau-parental reste aussi marginal : il contrevient à toute une représentation occidentale de la parenté. Ma chance a été d'échanger à ce moment-là avec Agnès Fine (1998) et Françoise-Romaine Ouellette (2001), qui au même moment travaillaient sur l'adoption, et en particulier la contestation montante de l'idéal de l'adoption plénière comme « nouvelle naissance » pour l'enfant, quitte à le couper de son histoire et de ses origines. Chacun sait que dans les représentations du lien de filiation, il y a une dimension biologique, une dimension sociale, et une dimension juridique et symbolique. Mais ce que nous avons compris, grâce à nos travaux sur ces situations marginales que sont l'adoption ou la famille recomposée, c'est que la véritable question rencontrée par les enfants n'était certainement pas l'alternative entre «le biologique » et «le social », mise en scène dans le débat public par ceux qui cherchent à définir le "vrai parent », mais bien plutôt un interdit social à conjuguer ces figures différentes.

Agnès Fine a élaboré la notion de « pluriparentalités » pour montrer que ce n’est pas au biologique ou au social, mais à la coexistence de diverses figures parentales que notre culture est intolérante (2002). Il y a des situations aujourd'hui où les géniteurs ne sont plus les parents (adoption), où les parents ne sont pas les géniteurs (procréation médicalement assistée [PMA] avec tiers donneur), où ceux qui s'occupent des enfants ne sont pas les parents du droit (familles recomposées), etc. Elle a montré que ces situations complexes restent marginalisées, comme s'il y avait un interdit les frappant, selon un principe d'exclusivité du parent. Pour compléter la réflexion, j’ai proposé de revenir vers notre système de parenté traditionnel en tenant compte de ce qu'il refoulait à ses marges, justement : les situations hors mariage, où l'enfant était un " bâtard » et où l'interdit de recherche en paternité (1789-1912) prouve bien que le modèle de référence n'était pas « biologique ». J'ai montré que nous sommes héritiers en réalité d'un système " matrimonial » selon lequel un « vrai parent » est celui qui cumule toutes les dimensions. Quand on admet cela, on comprend que les figures refoulées à la marge (mères sous X, parents de naissance des adoptés, donneurs et donneuses de gamètes, gestatrices dans les GPA [Gestation pour autrui], beaux-parents dans les familles recomposées, co-parents dans les familles homoparentales, etc.) sont toutes des traductions de l'angoisse sociale qui demeure sur ce que pourrait être un statut de parent qui ne serait pas « tout 
à la fois ». Les marginalisés sont en réalité tous des boucs émissaires de cette inquiétude sur le cœur de la norme.

Laurence Hérault : Mon travail sur l'expérience trans de la parenté et de l'engendrement s'inscrit également dans cette lignée de recherches, mais la parenté trans questionne autrement cette cohérence de la figure du parent. Elle interroge la dimension sexuée de cet empilement ordinaire des différents rôles assumés par le parent. La transition est une expérience qui transforme le faire famille et, réciproquement, la parenté a des effets sur les transitions. La parentalité et la transidentité ont été assez souvent perçues comme des expériences incompatibles ou difficilement compatibles à tel point qu'on a pu proposer, dans les années 1970, aux parents trans de se séparer de leurs enfants pour leur bien-être ou encore, jusque récemment en France, on a vu des équipes médicales refuser, a priori, de prendre en charge les personnes trans qui avaient des enfants. L'un des fondements de cette incompatibilité socialement perçue est l'apparente incohérence entre statut sexué et statut parental. Une femme trans, par exemple, qui a eu des enfants avant sa transition peut être définie et se définir à la fois comme père et comme femme, ce qui peut sembler a priori incohérent. Mais si l'on s'attache à décrire, par exemple, cette situation en termes d'identité narrative (Ricœur, 1991), cette incohérence apparente s'évanouit : elle s'est positionnée comme père depuis la naissance de son enfant et elle est désormais reconnue comme femme. Ce faisant, on devient capable de comprendre, de façon plus générale, qu'un statut parental ne renvoie pas uniformément à une inscription sexuée particulière, mais qu'il dépend tout autant d'une histoire familiale et des relations de parenté qui y ont pris forme. Dans les familles trans, comme dans les autres, être père ou mère est le résultat d'une histoire qui réfère aux dimensions tout à la fois corporelle, affective et sociale des relations interindividuelles. On peut être ainsi femme et père ou homme et mère sans qu'il y ait là de discordance identitaire mais simplement les effets d'un parcours de vie. Si l'on aborde également les expériences d'engendrement trans, celles des hommes qui accouchent ou celles des femmes qui procréent avec leur sperme par exemple, on est face à des réactions plus angoissées et violentes encore. Les hommes trans, notamment, qui mettent au monde leurs enfants sont ici une cible privilégiée, devenant des sortes de monstres qui bouleverseraient notre biologie et notre monde tout entier. Là encore si, pour décrire leur expérience on revient à leur histoire mais surtout à l'idée que l'engendrement est d'abord une question d'action plutôt qu'une question d'identité, l'incohérence affirmée s'évanouit également. Un homme trans qui accouche agit d'une certaine manière, manière qui est ordinairement celle des femmes, mais il s'inscrit en tant que père dans le registre des statuts du fait de sa reconnaissance en tant qu'homme. Cette situation permet de comprendre la différence entre statut dans la parenté et implication procréative. Cette différence invisibilisée dans les situations ordinaires, c'est justement les situations à la marge comme l'engendrement trans ou encore les situations de recours à la PMA ou à la GPA qui nous la rendent perceptible. 
Groupe EnCoRe : Pensez-vous que traiter de la marge comme objet pour éclairer la norme sociale puisse aussi amener à repenser les normes disciplinaires ?

Irène Théry : Pour moi, l'un des problèmes majeurs est la séparation anthropologie/sociologie, qui a l'air évidente pour tant de collègues, mais qui s'est faite seulement après la Deuxième Guerre mondiale. Avant, que ce soit Émile Durkheim, Marcel Mauss, Max Weber ou Norbert Elias, ils étaient aussi bien sociologues qu'anthropologues. La catastrophe de la coupure sociologie/anthropologie a été - en particulier dans le domaine de la parenté - que les anthropologues travaillaient sur « les autres » à partir d'un point de vue qu'ils n'interrogeaient pas, comme s'ils ne parlaient pas depuis un point de vue particulier. En un mot, ils ne se voyaient pas « autres parmi les autres». Et les sociologues travaillaient sur " nous aujourd'hui », sans bénéficier de la construction d'un " regard éloigné », ce qui les amenait, par exemple, à ne pas interroger la notion de famille nucléaire, qui leur paraissait aller de soi. Je crois qu'aujourd'hui dans nos travaux, quels qu'ils soient, on ne peut pas aborder la compréhension des marges ou des règles de nos sociétés sans les éclairer par la comparaison anthropologique, qui nous met nousmêmes en perspective. C'est la même chose pour la comparaison historique d'ailleurs. La famille nucléaire, ça semble tout simple : le père, la mère, les enfants. Et en fait c'est un énorme sujet. Si j'ai consacré sept ans à écrire La distinction de sexe (2007), c'est parce que je voulais mettre à plat tout ce que j'avais appris de l'anthropologie et de l'histoire sur les liens entre genre et parenté, et ôter son évidence à la notion de famille nucléaire qui s'élabore tardivement dans le système occidental de parenté, s'affirme avec le droit naturel moderne, et est au cœur des représentations naturalistes contemporaines des genres et des relations sexuées. Et toi, Laurence, dans tous les travaux que tu as faits sur toutes les formes de transidentités qui existent dans les sociétés, tu avais besoin du comparatisme pour aller réfléchir sur des comportements et des changements très actuels.

Laurence Hérault : C’est vrai aussi pour des gens comme Agnès Fine (p. ex. 2002), Jérôme Courduriès (p. ex. 2011), qui sont aussi dans notre univers de pensée. Même si nous travaillons sur nos sociétés, on a toujours l'idée qu'ailleurs ça se passe autrement, et donc en retour, que les questions qui sont posées ailleurs peuvent résonner ici. Quand j’ai commencé les travaux sur la transidentité, j’ai fait un terrain en France, dans une équipe médicale spécialisée. C'était un terrain passionnant et, parallèlement, j'ai travaillé sur la littérature de contact sur l'Amérique du Nord, pour essayer de saisir comment ces figures trans - appelons-les comme cela - sont pensées dans d'autres sociétés. Cela a été essentiel pour mettre en perspective la manière dont c'était pensé ici, pour « dépsychologiser » aussi l'abord de l'expérience trans. Voir comment on constitue un statut trans dans d'autres sociétés, c'est fondamental pour moi. Cette dimension-là, même si elle ne se donne pas à voir dans des terrains comme on l'attendrait en anthropologie, elle se donne à voir dans la manière dont j'essaie d'aborder la question. Il y a aussi ce retour à la dimension historique qui est importante pour comprendre 
comment s'est construite la compréhension de la transidentité dans notre société. Cela permet de voir ce que le modèle pathologique a permis mais aussi de comprendre comment il a pu enfermer les vies trans dans une conception problématique. Autrement dit, cette perspective comparative accroît ou oblige à être sensible aux diverses modalités de description des situations et des expériences. Si je reprends l'exemple de l'engendrement trans, on voit bien que décrire cette expérience en termes d'incohérence identitaire ou au contraire en termes d'action et de relation modifie sa compréhension et peut donc avoir des effets sur l'acceptabilité sociale.

Groupe EnCoRe : Nous comprenons de vos deux propos que les outils de la description ne sont pas toujours adaptés à la description des marges, ce qui oblige à repenser nos outils et nos méthodes descriptives. Dans cette optique, quels seraient les outils de la description?

Laurence Hérault : Si on prend l'exemple de l'engendrement trans, il a la particularité de mettre au centre la dimension de la sexuation des personnes. Le cas célèbre de Thomas Beatie ${ }^{4}$ est souvent décrit comme une impossibilité logique : un homme n'accouche pas, donc Thomas Beatie ne peut pas être un homme. Il ne faut pas oublier qu'il y a des médecins qui ont qualifié les parents trans de " chimères parentales "! Alors, comment sortir de la confusion et de ce désordre ? En fait le trouble vient du fait qu'on reste coincés sur les questions des propriétés et de l'identité, d'une conception dualiste de la personne qui sépare en deux entités « le corps » et « l'esprit» (ou « le moi »), qui sépare le corps de l'expérience vécue. Le rapport de l’Académie de médecine sur l'autoconservation des gamètes des personnes trans ${ }^{5}$ en est le parfait exemple. On y décrit des identités multiples pour les personnes trans : une identité parentale, une identité génétique, une identité reproductive. Tout ça pour démontrer que les personnes trans qui engendrent avec leur corps sont des chimères ! Mais si on fait un pas de côté, si on remet le corps là où il est, si on remet la temporalité, les actions et les relations - toutes choses qui sont déjà là !-, et bien « la vision chimérique » tombe. On n’invente rien, on ne fait que décrire autrement une situation : on n'a pas besoin de changer de biologie pour comprendre qu'un homme trans est en capacité d'accoucher puisqu'il a un utérus. L'expérience trans de la parentalité n'est pas dissociable de la temporalité, des relations, et c'est de cela dont on doit rendre compte dans nos descriptions : agir à la manière de, agir en tant que, c'est l'outil descriptif que j’ai proposé. Thomas Beatie agit à la manière des femmes, et il agit en tant que père ; c'est-à-dire qu'il met son statut dans l'ordre de la parenté en cohérence avec ses autres statuts, donc il a un statut de père bien qu'il accouche de son enfant.

\footnotetext{
${ }^{4}$ Thomas Beatie est un homme trans américain qui a porté et mis au monde son enfant (voir Hérault, 2011).

5 Pierre Jouannet, Académie de Médecine (2014), Autoconservation des gamètes de personnes transsexuelles et projet parental éventuel. En ligne, consulté le 24/10/2019. URL : http://www.academie-medecine.fr/wp-content/uploads/2014/03/25.3.14-JOUANNET-info2.pdf
} 
Irène Théry: On résout énormément de faux problèmes lorsqu'on passe d'une socio-anthropologie des identités et des classes ontologiques - ce que Cornelius Castoriadis appelait notre tendance à voir le social de façon « ensembliste-identitaire » (1975) - à une socio-anthropologie de l'action. L'agir humain est singulier, et c'est ainsi que j'ai tenté de montrer, à partir de M. Mauss, qu'on peut penser le genre non pas comme une propriété des personnes, mais comme une manière d'agir référée à des attentes sociales. Dans cette perspective, le genre est adverbial, relatif et relationnel tout à la fois (Théry, 2007). Les problèmes qui sont posés comme des questions ontologiques de propriétés et d'attributs, qui donnent des sortes de classifications des êtres par exemple, ces problèmes se résolvent dès lors qu'on aperçoit que les êtres humains vivent, c'est-à-dire agissent, les uns en relation avec les autres, et en référence à des systèmes de règles, de représentations, de valeurs. Chez les humains, on n'est pas guidé par un « moi » qui serait une sorte de moteur intérieur, mais on a une capacité particulière à " désadhérer » de soi-même, à se regarder soi-même, ce qui permet de mettre à distance sa propre expérience, de la complexifier.

Laurence Hérault : L'expérience transgenre c'est une façon d'agir, de vouloir agir en tant que ceci, ou en tant que cela, et pas d'être ceci ou cela. Et les gens aussi bougent, c'est ça qui est intéressant et complexe à décrire parfois. C'est pour ça qu'il faut remettre la question de la temporalité, par exemple pour décrire comment on peut être le père de son enfant alors qu'on est une femme. Pour ça, il faut déplier l'histoire : on a eu un enfant avant sa transition, donc on a été son père; et maintenant on est une femme. Ces identités a priori contradictoires, en fait, ne le sont pas, si on remet l'histoire au milieu. On a été un père, et comme on s'est institué comme père auprès d'un enfant, comme l'enfant vous a mis aussi dans cette position-là et que socialement aussi vous avez été défini comme cela, on peut peut-être continuer à faire exister cette relation-là (être père), avec une autre relation qui semble a priori contradictoire (être femme). Mais cela peut se modifier au fil du temps, au fil de l'expérimentation commune d'une relation que les protagonistes vont souhaiter requalifier. Si on en revient aux actions et aux relations, alors la question du corps peut être pensée d'emblée parce que les relations aux autres, elles se font nécessairement par la médiation du corps. Et si on reprend des représentations qu'a bien travaillées Marilyn Strathern (1988), comme la matérialité corporelle, elle montre que le corps est le produit des relations. Dans cette approche-là, le corps n'est jamais de côté, jamais oublié. Il est toujours au centre parce que le corps est produit par les liens et il est le produit du lien.

On est dans des espaces - la transidentité, la procréation - où le corps est essentiel. On ne peut pas le mettre de côté puisque c'est précisément le cœur de la question. Mais à condition de penser que le corps ne se réduit pas au biologique. Parce que les objets auxquels on s'est affrontées nous posaient problème, parce qu'on était aux prises avec ces conceptions dualistes de la personne, on a été obligées de repenser autrement la question du corps. 
Irène Théry: Laurence, je me souviens combien tu m’as libérée de problèmes insolubles en forgeant des outils pour décrire l'expérience de Thomas Beatie : un homme peut agir en tant que «la mère porteuse » de son propre couple, comme il le dit lui-même. Là, il y a des passages de frontières qui sont bien plus près du vécu social des personnes, bien plus intéressants que les théories qui s'imaginent que pour penser l'égalité des sexes et des sexualités il faudrait ne plus voir que des «moi », des volontés sans corps sexué, sans corps du tout, à la limite, comme on peut le voir aujourd'hui dans le cadre par exemple des débats bioéthiques dans les controverses sur la filiation, avec l'obsession de certains de se débarrasser de toute référence au corps, au sexe et au genre.

Laurence Hérault : De toute façon, les gens se réfèrent quand même à ces catégories : masculin ou féminin. Elles restent opérantes. Ce qui est difficile dans l'approche relationnelle que nous utilisons, c'est le "pas de côté » qu'elle suppose, et ce "pas de côté » que nous avons fait n'est pas toujours compris en tant que tel. Cette approche est pourtant très utile dans ces situations complexes où l'on veut rendre compte de l'expérience des gens : par exemple, une femme trans qui est en couple avec une femme cis. Le couple s'est formé avant sa transition, et elles ont eu deux enfants au cours de leur mariage, puis un troisième qui arrive après la transition. Ce qui est intéressant, c'est que cette femme trans revendique le lien de filiation avec ce troisième enfant, qu'elle a conçu avec ses spermatozoïdes comme les deux précédents, mais on lui refuse cette filiation au titre que l'on ne peut pas avoir deux mères à l'état civil. On lui a proposé juridiquement d'adopter son enfant, comme le fait n'importe quel couple de lesbiennes. Quand j'ai fait un entretien avec cette femme et sa compagne, j'ai compris que le cœur de cette revendication c'était de dire : « je ne peux pas établir mon lien de filiation avec ma fille par l'adoption parce que ça créerait une différence avec mes autres enfants où la filiation a été établie autrement. Et il n'y a pas de raison. Je ne veux pas qu'il y ait de différence entre mes enfants parce que mes enfants je les ai tous mis au monde de la même façon ». Et on voit bien que derrière cela, ce qui importe, c'est de mettre en relation, de revendiquer une capacité à agir, à agir d'une certaine manière et pas d'une autre.

Irène Théry : Et juste une question : est-ce qu'elle se vit comme la mère ou toujours comme le père des premiers enfants?

Laurence Hérault : Je pense que c'est en train de bouger. Et c'est cela qui est intéressant, c'est-à-dire que dans le premier entretien que j'avais fait il y a environ quatre ans, elle se disait le père de ses enfants en tant que femme. Cette année, j'ai eu un nouvel entretien, après un jugement qui a établi qu'elle est le "parent biologique » de son enfant. Cette reconnaissance de son lien de filiation est importante car elle lève l'insécurité liée au refus de l'inscrire sur l'état civil de sa fille ; mais la désignation comme « parent biologique » qui n'existe pas en droit français, et qui n'est pas en adéquation avec la manière dont elle vit sa relation avec sa fille, n'est pas satisfaisante. Elle ne veut pas être ainsi marginalisée, comme elle le dit, elle ne veut pas être un «parent biologique ». Elle ne 
veut pas être le seul « parent biologique » désigné à l'état civil français [à l'état civil on est père ou mère]. On arrive donc à une situation paradoxale où au moment même où l'on accepte de reconnaître son lien de filiation, on le fait d'une manière qui la spécifie voire la vulnérabilise socialement.

Laurence Hérault : L'expérience de cette famille est aussi la preuve qu'on doit toujours prendre en considération la dimension de la temporalité : les choses ne sont pas figées une fois pour toutes. Tout cela se constitue dans le temps, à travers le temps. Il y aurait de belles enquêtes à faire là : comment on passe à un moment donné, temporellement, d'un statut à l'autre ? C'est-à-dire comment un enfant peut vous désigner à un moment comme une mère alors que vous avez été son père ? Et avec peut-être une période de transition où il vous a désignée et comprise comme une femme qui était son père. Tout ce travail, il faut le faire sur le terrain, on ne va pas le décider là, comme cela, théoriquement. Pour cela, il faut aller voir les gens, et il faut juste essayer de décrire comment ils ont bougé, comment ils changent. Ce serait intéressant de voir là quels sont les seuils, quels sont les moments où quelque chose comme ça peut arriver. Ce couple dont je parle c'est une situation pour l'instant encore exceptionnelle : il y a des enfants avant la transition, il y a des enfants après et avec le même couple. Il y a cette tension très particulière de la temporalité, où quelque chose va se jouer autour de la filiation : je veux que cette filiation soit établie comme les deux précédentes, mais je veux que mon statut au quotidien soit établi sur le présent. Donc cela suppose que des choses qui avaient été pensées d'une certaine manière au départ vont aussi être amenées à évoluer. C'est pour cela qu'il faut « attraper » l'expérience des gens et essayer simplement de la décrire, dans toute sa complexité, sans la stigmatiser ni la spécifier abusivement.

Groupe EnCoRe : Laurence, vous parliez de seuils et de temporalité. On pense tout de suite à l'analyse des rites de passage d'Arnold van Gennep (1991), repris par la suite par Victor Turner (1990). Les outils analytiques que ces deux auteurs proposent sont-ils utiles pour rendre compte de l'expérience trans?

Laurence Hérault : La marge, dans le schéma de van Gennep, c'est une étape dans un processus de passage d'un statut vers un autre statut. Anne Bolin (1987) utilise ce schéma pour saisir la transition, quand elle dit par exemple qu'on passe d'une identité d'homme à une identité de femme. La transition en elle-même, c'est la période de marge, et elle l'étend, comme van Gennep le fait parfois, en disant que la marge peut être autonome. Mais ce qu'elle dit aussi au fond, c'est que le statut d'avant cette période de marge, c'était la confusion de genre. On passerait donc de la confusion à une identité véritablement définie, comme celle de femme trans. Le problème, c'est que, pensée de cette manière, la transition devient un rite thérapeutique. Bolin reprend les dispositifs médicaux. La transition guérit quelque chose, ou du moins elle permet de sortir d'un trouble. C'est une bonne traduction de la conception classique occidentale de la transition. Mais cette approche est problématique parce qu'un certain nombre 
d'expériences trans - qui sont peut-être plus visibles aujourd'hui que dans les années 1980 - y échappent. Cela ne permet pas de penser la transition en dehors de la résolution d'un trouble ou d'une confusion. Donc ce n'est pas satisfaisant. De plus, la transition est un peu essentialisée selon moi. Elle est vue comme un rite de passage ; c'est une métamorphose de l'identité personnelle. C'est ce qu'Anne Bolin dit. C'est vraiment comme si l'individu se transformait de part en part, comme s'il n'était plus du tout ce qu'il était auparavant. Bolin modifie peut-être là l'idée de départ de van Gennep qui, lui, pose la question plus sociologique des statuts. Bolin en fait une question ontologique. L’identité complète de la personne serait transformée. Encore une fois cela correspond à la conception classique occidentale mais cela pose problème parce qu'un certain nombre d'expériences trans y échappent. Il y a donc un paradoxe dans cet usage des rites de passage. La temporalité est de plus réduite à une question d'avant/après. C'est une dimension centrale dans les rites de passage et, ici, elle est réduite à deux temps disjoints qui sont l'avant et l'après. C'est un autre problème de l'approche d'Anne Bolin. Le cadre rituel contraint les actes. Il définit un certain nombre d'actions qui doivent être accomplies d'une certaine manière et dans une certaine temporalité pour obtenir le résultat attendu. Si celui-ci n'est pas atteint, c'est que l'on n'a pas agi comme on aurait dû agir. Cette perspective ne permet pas de bien décrire le processus de transition, qui est toujours ouvert. Les rôles ne sont pas définis. Les personnes peuvent coopérer avec les médecins ou s'y opposer. La situation d'arrivée non plus n'est pas prédéfinie. Il y a toujours une dimension d'incertitude. Le rituel, au contraire, est un dispositif qui vise à réduire l'incertitude. La liminarité au sens de Turner est donc plus souple parce qu'il la sort du cadre rituel. Pour lui, l'expérience liminaire peut être une expérience au long court (Turner, 1990) ; elle peut se stabiliser. Il donne l'exemple de l'engagement religieux des moines. Un certain nombre de chercheurs ont repris cette idée pour penser, non pas la transition, mais l'expérience trans dans d'autres sociétés. Niko Besnier et Alexa Alexeyeff (2016) reprennent par exemple cette idée de liminarité pour penser l'expérience trans en Océanie, où la conception occidentale d'une métamorphose identitaire dans la transition est effectivement absente. La liminarité dans le sens de V. Turner est donc sans doute utile. Mais, de fait, je fais peu d'usage de ces deux notions. Je reprends plutôt la version de la condensation rituelle de Michael Houseman et Carlo Severi (1994) qui ont repris et prolongé l'analyse de Gregory Bateson sur les rituels de travestissement iatmuls (1971). Dans leur idée, le travail rituel ne consiste pas à modifier l'identité personnelle mais plutôt à modifier les relations et donc à produire de nouvelles positions sociales et de nouvelles possibilités d'action et de relation.

Groupe EnCoRe : On s'éloigne donc de la conception identitaire qui pose problème dans l'interprétation d'A. Bolin et on revient à l'importance des actions et des intentions dont nous parlions précédemment.

Laurence Hérault : C'est ça. Il est aussi question des statuts parce que modifier les relations, cela implique de repositionner les personnes les unes par rapport aux autres. 
Mais on approche le statut par la relation au lieu d'y entrer par une dimension identitaire plus rigide. Les actions et les relations sont centrales dans leur approche. Le rituel actualise des modalités d'actions qui normalement sont contradictoires. Par exemple, certains rituels de travestissement iatmuls modifient les positions de personnes qui vont se trouver dans une position à la fois d'ascendance et de descendance vis-à-vis des mêmes individus : c'est impossible. Si l'on est le descendant de ses parents, on ne peut pas être leur ascendant. Les rituels de travestissement mettent cette situation en scène. En actualisant ces modalités contradictoires, ils modifient les relations, ce qui va aussi avoir des effets sur les relations plus quotidiennes. Les rituels permettent par exemple d'inscrire une relation avec l'oncle maternel dans des systèmes où la filiation patrilinéaire ne la prévoit pas. Cela crée du mouvement. Cette perspective m’intéresse particulièrement pour penser l'engendrement trans. Dans la situation d'un homme enceint ou dans celle d'une femme trans qui procrée avec ses spermatozoïdes et qui serait donc en position de père, on voit a priori quelque chose de contradictoire, c'està-dire qu'on ne pourrait pas être père et accoucher de son enfant (Hérault, 2011). On peut interpréter cela comme un brouillage, une confusion, ou bien, justement, on peut plutôt essayer de montrer que l'engendrement n'est pas une question de propriétés personnelles. C'est une question de relations et de positionnement dans un réseau de relations. Ces relations ne sont peut-être pas aussi contradictoires que l'on pourrait le penser dès lors qu'on déplie l'engendrement. Il s'agit de montrer les engagements particuliers des personnes, de rendre compte de leurs actions et des relations en jeu plutôt que d'en faire une question d'identité et de propriétés personnelles individuelles.

Groupe EnCoRe : Une autre autrice majeure qui aborde la question des marges, c'est Mary Douglas (2001). La notion de temporalité semble moins présente dans son approche. En tous cas, elle aborde moins la question des relations qui se modifient que celle du système normatif. Comment entre-t-elle en résonance avec ces notions plus temporelles et processuelles?

Irène Théry : La lecture de Mary Douglas a été une découverte majeure pour moi sur ces questions. Mais pour l'expliquer, je dois d'abord revenir à Mauss. En travaillant sur la parenté en rapport avec la question du genre, sa conférence intitulée « La cohésion sociale dans les sociétés polysegmentaires » (1981) m’a particulièrement marquée. C'est dans ce texte qu'il écrit : "Nous n'avons fait que la sociologie des hommes et non pas la sociologie des femmes, ou des deux sexes »(1981:15). Il prononce cette phrase dans un contexte particulier où domine la thèse durkheimienne opposant la " solidarité organique » moderne à la " solidarité mécanique » des sociétés qu'on nommait alors primitives (Durkheim, 2013). Mauss remet en cause la notion même de solidarité mécanique. L'ethnologie a montré que les sociétés les plus éloignées des nôtres, les sociétés sans État ou sans écriture par exemple, sont extrêmement organisées. Il souligne l'existence de quatre distinctions statutaires, plus prégnantes que partout ailleurs : par sexes, par âges, par générations et par clans. Ces distinctions se combinent dans les 
statuts que les personnes endossent pour agir selon des règles, et constituent le lien de la société. Dans De la souillure, Douglas reprend la description de la dramaturgie sociale complexe organisée par ces systèmes de distinctions/relations, et met au centre de sa réflexion les frontières internes et externes d'une société (2001). Son grand apport est de centrer sa réflexion sur les interdits et les inquiétudes causées par ces frontières, ces limites et les sanctions qui accompagnent leur éventuel franchissement. En tant que telle, son étude des confins et des marges est décisive. Mais Douglas va plus loin. Elle montre que les distinctions et oppositions qui organisent la société - auxquelles Claude Lévi-Strauss s'est beaucoup intéressé pour comprendre les cosmologies - ne sont pas seulement des modes de représentations du monde, mais d'abord et avant tout des organisateurs de l'action. Elles permettent de poser la question du pouvoir et de l'autorité ; la lecture de Douglas par Vincent Descombes dans Proust, philosophie du roman (1987) est décisive sur ce point : elle met en exergue l'importance capitale de distinguer ces deux notions, en général considérées comme synonymes. Le pouvoir pose la question de savoir pourquoi un événement, comme la mort d'un troupeau de bétail, est survenu, et quelle puissance l'a provoqué. Les êtres considérés comme marginaux s'en voient souvent imputer la responsabilité au titre des pouvoirs extraordinaires qui leur sont prêtés. L'autorité est très différente. Elle concerne les droits et des devoirs : à qui puisje demander de l'aide ? que puis-je attendre de quelqu'un ? Bref l'autorité renvoie à ce que chacun peut et doit faire selon son statut. Cette distinction est majeure pour comprendre, par exemple, qu'avec l'avènement de l'égalité des sexes comme valeur cardinale de la démocratie, nous n'avons pas affaire seulement à une contestation du " pouvoir » des « dominants » (blancs, masculins, hétérosexuels, cisgenre, etc.) comme on le croit dans une perspective ensembliste-identitaire, mais à une métamorphose de tout le système normatif commun (représentations, règles, valeurs, normes) faisant autorité et définissant les autorités.

Groupe EnCoRe: Vous défendez donc l'une et l'autre une approche relationnelle et temporelle de l'agir humain, qui se distingue d'une socio-anthropologie des états ontologiques. Dans votre approche, pour produire des descriptions de sciences sociales, il faut replacer la succession des actions dans la dimension du sens et du temps, autrement dit, dans les "systèmes d'attente institués » dirait Mauss (1950). Est-ce que cela n'implique pas aussi un travail sur nos catégories de pensées, celles qui servent à décrire les actions?

Irène Théry : Nous nous pensons tous avec les catégories que nous avons à notre disposition, que notre société et notre temps nous proposent, mais parfois ces catégories conviennent mal à la façon dont on expérimente les choses. Travaillant depuis près de vingt ans sur la PMA avec tiers donneur, j'ai peu à peu perçu l'écart immense entre d'une part les catégories proposées pour parler du don, et d'autre part l'expérience des personnes qui sollicitaient et recevaient ce don (Théry, 2010). À l'époque, les catégories pour penser le don avaient été entièrement constituées par et pour les médecins. Pour eux, c'était simple, ils rendaient possible une procréation en utilisant des gamètes fonc- 
tionnels qui leur avaient été fournis, comme ils auraient pu utiliser d'autres « produits du corps humain ». Dans cette vision biomédicale, le don n'est possible que si le parent n'est pas biologique, bien que le modèle de référence soit la procréation. Du coup, la solution est de cacher le don pour que le parent stérile passe quand même pour un géniteur.

Or dès qu'on s'intéressait à l'expérience des parents, la particularité de leur situation était justement que pour un même projet, pour un même enfant, on n'a pas une opposition mais au contraire une alliance du parent biologique et du parent social. Pour le couple qui reçoit un don, cela fait sens et valeur de procréer un enfant, et cela fait aussi sens et valeur de pouvoir avoir un enfant sans procréer. Comment rendre compte de cet écart entre l'expérience des personnes et un modèle bioéthique centré sur une vision biomédicale très instrumentale. Je pense que mon apport principal sur ce sujet tient en quelques pages de Des humains comme les autres (2010). J'ai mis en œuvre ce que j'avais appris d'un point de vue théorique en travaillant avec Vincent Descombes sur l'action humaine complexe (1997) - ce que c'est que participer à une action complexe - en disant : en fait un don, c'est une action humaine complexe que l'on peut nommer « l'engendrement avec un tiers donneur ». Cette action complexe est une seule action; elle est longue, elle est compliquée. Qui y participe ? Un couple, où l'un procrée et l'autre pas ; une tierce personne, donneur ou donneuse qui va donner de sa capacité procréatrice pour que d'autres soient parents, le tout avec la médiation de professionnels. Une action dont la particularité est que son but est la naissance d'un enfant, qui est ainsi un partenaire potentiel de l'engendrement, ce dont ne parle jamais le modèle biomédical qui copie le « don de gamètes » sur le « don de sang ». Il a fallu que je m’asseye à ma table, que je fasse des petits schémas pour commencer à décrire les choses, sans jamais oublier que les gens agissent avec des intentions, dans un contexte institué. D’un coup il m'a sauté aux yeux que ce que la vision médicale empêchait de décrire, c'est tout simplement la coopération entre donneurs et receveurs.

Groupe EnCoRe : Une dernière question pour conclure. Y-a-t-il des sujets qui vous paraissent importants à étudier à l'avenir pour éclairer ces notions de marges, frontières, seuils, confins?

Irène Théry : Sur la question des confins, je pense qu'il y a encore énormément à faire, tant sur les confins du début et de la fin de vie. Anne-Sophie Giraud a énormément apporté avec sa thèse sur l'être anténatal, mettant la temporalité et le changement au cœur de son étude des confins de la conception et de la naissance (2015). On ne prend pas encore toute la dimension de ce qu'un tel travail ouvre comme perspectives sur notre vision des statuts successifs de l'être anténatal et des principes qui organisent cette succession.

Sur le sujet de l'agonie, je me suis également trouvée avec le même terme de confins. J'ai publié un article sur le sujet (Théry, 2012), basé sur une très longue enquête auprès de 150 personnes séropositives, sur leurs silences, secrets, confidences dans leurs rap- 
ports aux proches. Il y a une dimension relationnelle de l'agonie. Les gens très malades tiennent à ce que les statuts ne soient pas bouleversés, que ma femme ne devienne pas mon infirmière, que mon enfant ne devienne pas mon parent. En même temps, il y a aussi un moment où on passe de la maladie à l'agonie, et où on arrive aux confins : alors ces statuts s'abolissent d'une certaine manière, car, cette fois, la seule tâche humaine est d'accompagner celui qui est encore parmi nous et déjà plus parmi nous. Ce moment est tragique, et sa dimension relationnelle encore peu reconnue. Il y a beaucoup de travaux à mener qui compléteraient, en miroir, ce qu'elle a fait sur la naissance.

Laurence Hérault : Je pense à toutes les expériences ou tous les dispositifs qui permettent d'explorer qui peut être parent dans une société. C'est peut-être moins le cas en assistance médicale à la procréation, mais pour la question trans c'est assez évident. Il y a des personnes qui sont très légitimes pour revendiquer le statut de parent et d'autres qui ne le sont pas. Il faudrait étudier tous les lieux où cette question est implicitement posée car cela rejoint aussi la question de la marge et de l'exclusion. On voit bien aujourd'hui qu'exclure de la parenté, c'est aussi exclure socialement. Mais on ne peut pas détacher la question du corps de ce qu'il se passe dans la parenté, donc je pense évidemment aussi aux questions d'auto-préservation de la fertilité des personnes trans, ainsi qu'à la question de la contraception et des stérilisations. Tous les lieux où on peut tenir le corps et la parenté, le corps et les relations...

Groupe EnCoRe: Nous vous remercions infiniment pour vos remarques et réflexions éclairantes pour le sujet du numéro.

\section{Bibliographie}

BATESON G. (1971), La cérémonie du Naven. Les problèmes posés par la description sous trois rapports d’une tribu de Nouvelle-Guinée, Paris, Les Éditions de Minuit.

Besnier N., AleXeyefF K. (2016), Gender on the edge: Transgender, gay, and other Pacific Islanders, Honolulu, University of Hawai'i Press.

Bolin A. (1987), In search of Eve. Transsexual rites of passage, Londres, Bergin and Garvey.

CASTORIADIS C. (1975), L'institution imaginaire de la société, Paris, Seuil.

CoURduriès J. (2011), Être en couple (gay). Conjugalité et homosexualité masculine en France, Lyon, Presses universitaires de Lyon.

Descombes V. (1987), Proust. Philosophie du roman, Paris, Les Éditions de Minuit.

Descombes V. (1997), Les institutions du sens, Paris, Les Éditions de Minuit.

Domat J. (1689), Les lois civiles dans leur ordre naturel, Paris, J.-B. Coignard.

Douglas M. (2001 [1966]), De la souillure. Essai sur les notions de pollution et de tabou, Paris, La Découverte. 


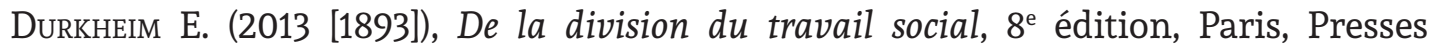
universitaires de France.

Fine A. (1998), Adoptions. Ethnologie des parentés choisies, Paris, Éditions de la Maison des sciences de l'homme.

Fine A. (2002), «Qu'est-ce qu'un parent ? Pluriparentalités, genre et système de filiation dans les sociétés occidentales », Spirale, 2002, vol. 1, n²1, p. 19-43.

Gennep A. (van) (1991 [1909]), Les rites de passage. Étude systématique des rites, Paris, Picard.

GIRAUD A-S. (2015), Les statuts de l'être anténatal: un processus d'humanisation «relationnel». Assistance médicale à la procréation et mort périnatale, thèse de doctorat, École des Hautes Études en Sciences Sociales.

HÉRAULt L. (1996), La grande communion. Transformations et actualité d’une cérémonie catholique en Vendée, Paris, Éditions du CTHS.

HÉRAult L. (2011), « Le mari enceint : construction familiale et disposition corporelle », Critique, 2011, vol. 764-765, $\mathrm{n}^{\circ}$ 1, p. 48-60.

HÉRAult L. (dir.) (2014), La parenté transgenre, Aix-en-Provence, Presses universitaires de Provence.

Houseman M., SeVeri C. (1994), Naven ou Le donner à voir. Essai d'interprétation de l'action rituelle, Paris, Éditions de la maison des sciences de l'homme.

Mauss M. (1950), Sociologie et anthropologie, Paris, Presses universitaires de France.

Mauss M. (1981), « La cohésion sociale dans les sociétés polysegmentaires », in CEuvres, vol. III, Paris, Les Éditions de Minuit, p. 11-27.

Ouellette F-R. (2001), " L'intérêt de l'enfant adopté et la protection de ses droits », Éthique publique, vol. 3, n 1 . En ligne, consulté le 24/10/2019. URL : http://journals. openedition.org/ethiquepublique/2645.

Riceur P. (1991), Temps et récit. Paris, Seuil.

StRATHERn M. (1988), The gender of the gift: Problems with women and problems with society in Melanesia, Berkeley, University of California Press.

THÉRY I. (1983), La référence à l'intérêt de l'enfant dans les procédures de divorces. Usage judiciaire et ambiguïtés, thèse de doctorat, Paris, Université Paris V.

THÉRy I. (2007), La distinction de sexe. Une nouvelle approche de l'égalité, Paris, Odile Jacob.

THÉRY I. (2010), Des humains comme les autres. Bioéthique, anonymat et genre du don, Paris, Éditions de l'EHESS.

THÉRY I. (2012), « L'agonisant et le triangle des proches. Pour une approche relationnelle de la fin de vie », La vie des idées. En ligne, consulté le 24/10/2019. URL : https:// laviedesidees.fr/L-agonisant-et-le-triangle-des.html.

TuRner V. (1990 [1977]), Le phénomène rituel : structure et contre-structure, Paris, Presses universitaires de France. 